\title{
LA VIDA ÚTIL DE UN ACTIVO Y POLÍTICA DE REEMPLAZO DE ACTIVOS*
}

\author{
Sergio Bravo Orellana \\ PROFESOR AUXILIAR DE ESAN \\ Área de FinanZas, Contabilidad y Economía
}

Con la colaboración de:

Diego Cueto Saco

ASISTENTE ACADÉMICO

\section{Resumen}

En evaluación de proyectos de inversión, un aspecto importante es la determinación de la vida útil de activos para su utilización en temas como el reemplazo de activos o la perpetuidad de las inversiones. Este texto pretende desarrollar el concepto de "vida útil económica de un activo" a través de definiciones y ejemplos ilustrativos. Se establece el cálculo detallado de costo anual equivalente para determinar la vida útil económica de un activo.

\section{Introducción: vida útil física $y$} vida útil económica

Uno de los primeros puntos por desarrollar es el deslinde entre los conceptos de 'vida útil física' y 'vida útil económica'; esta diferenciación tiene como contexto el análisis del caso de una plantación de naranjos.

* Quienes estén interesados en los conceptos vertidos en un modelo financiero pueden consultar la página web: http://www.esan.edu.pe/sbravo/ sbravo.htm, donde se encuentra la hoja de cálculo respectiva.
Para iniciar una plantación, se deben realizar inversiones en el cultivo y crecimiento de la plantación hasta tener los primeros frutos susceptibles de ser comercializados al final del tercer año ${ }^{1}$. Para los primeros tres años, la producción, generalmente, no es comercializada. Por lo mismo, se considera a este periodo como

1 Las características de la plantación varían dependiendo de la zona donde esté ubicada; en ese sentido, los datos que se registran aquí sólo deben ser tomados como referenciales. 
la etapa preoperativa, hasta que la producción puede ser vendida al mercado. Cuando se posee producción que se puede vender, se inicia un proceso de incremento significativo de la productividad. En el caso concreto al que se refiere este ejemplo, la productividad llega a las 8 toneladas por hectárea en el año 7 (8 t/ha); 32 t/ha en el año 15; 38 t/ha en el año 20. A partir de este año, se estabiliza el crecimiento y, luego, empieza a descender hasta llegar a las 28 t/ha en el año 25 y 12 t/ha en el año 30. En la Figura 1 se observa que la plantación puede dar frutos más allá del año 40, pero con productividades muy bajas inferiores a 4 t/ha.

Figura 1. Vida útil económica de una plantación

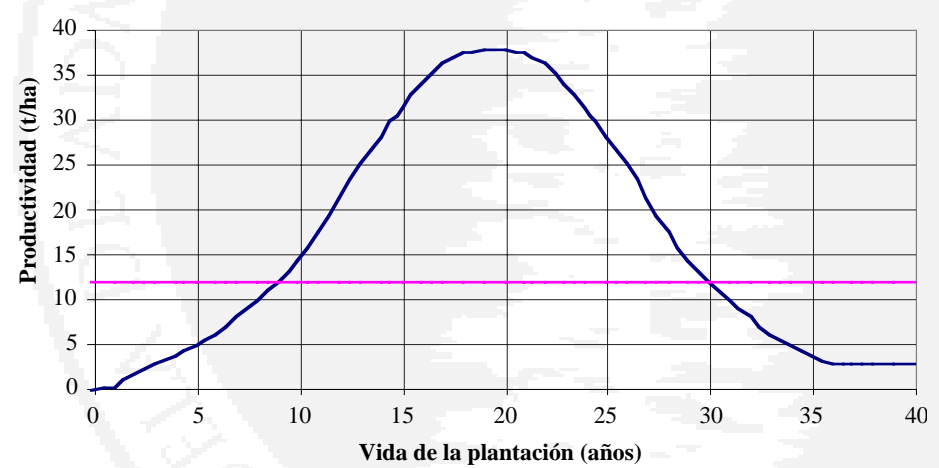

una línea recta, el punto de equilibrio, cuya productividad es $12 \mathrm{t} / \mathrm{ha}$.

Se percibe, entonces, que la plantación puede vivir, 'vegetativamente', hasta pasados los 50 años; este periodo vital es lo que se denomina 'vida útil física' del activo (en este caso, la plantación). Sin embargo, en términos económicos no es rentable seguir costeando los egresos de sostenimiento de la plantación, pues la productividad es baja, tanto que lo racionalmente económico es realizar un proceso de 'quemado' de la plantación para reemplazarla con una nueva.

En consecuencia, la vida útil económica se prolonga hasta cuando, económicamente, es justificable sostener el activo. En el caso del ejemplo, la vida útil económica se limita al año 30, pues los ingresos aportan positivamente sólo hasta ese año.

Un análisis más fino del costo de oportunidad supone la utilización del terreno en un nuevo cultivo a partir de los años finales de la plantación. En ese sentido, el reemplazo de la plantación puede ser, inclusive, antes de lo señalado. La forma de hacerlo se deriva de los conceptos que se presentan a continuación.

\section{El concepto de vida útil económica}

El activo que sustenta la producción de un bien o servicio posee una vida útil como tal; es decir, llega el momento en que, 
por desgaste, no puede seguir siendo utilizado para los fines que fue fabricado o adquirido. Como ya ha sido dicho aquí, puede ser inclusive económicamente más conveniente retirarlo de los activos de la empresa antes de ese momento.

La vida útil se puede prolongar (o acortar) aumentando (o disminuyendo) los gastos de mantenimiento. A pesar de esto, no es imprescindible que el activo llegue al término de su vida útil como tal, inclusive en la situación de que el activo se halle óptimamente mantenido; por razones económicas, se puede dejar de utilizar el activo. El periodo durante el cual se debe utilizar el activo atendiendo a razones económicas se denomina 'vida útil económica' del activo.

Para ensayar una definición más completa de vida útil económica de un activo, se debe distinguir entre aquellos activos que están ligados directamente a los ingresos - como es el caso de una plantación$\mathrm{y}$ aquellos activos fijos que forman parte del parque de la maquinaria o de los equipos de la empresa, sin estar íntimamente ligados a los ingresos. Sobre la base de esta diferenciación, se puede establecer lo siguiente:

a) La vida útil económica de activos relacionados con ingresos es aquella que se determina por el tiempo transcurrido hasta que la utilidad económica llega a ser cero. Durante este periodo, los ingresos generados por el activo cubren todos los costos económicos de la empresa, lo que incluye los costos de operación y mantenimiento, y los costos del capital invertido.

b) La vida útil económica de activos relacionados con costos es aquella que se determina por el tiempo en el que el costo total del activo referido al periodo de reemplazo es el menor de todos. Durante este periodo, los costos económicos que corresponden al activo son marginalmente menores que cualquier alternativa de periodo de permanencia del activo dentro del negocio. Si se cumple este precepto, la empresa puede generar los mayores excedentes por tener costos de activos menores. Este caso se analiza con mayor detenimiento en el siguiente acápite.

\section{Determinación de la vida útil económica}

El siguiente ejemplo sirve para determinar por cuanto tiempo se debe conservar un activo en la empresa, con la finalidad de que se eviten costos innecesarios de operación y mantenimiento. Es importante recordar que la vida útil económica del activo difiere, significativamente, de lo que es la vida contable o vida útil física propiamente dicha. Los conceptos que se presentan a continuación son aplicables en el caso de activos que no están directamente relacionados con los ingresos del negocio, como puede ser un vehículo de transporte dentro de una flota de vehículos o un activo en general dentro de un parque de activos.

\subsection{El concepto de costo total de una decisión de inversión}

La racionalidad económica aconseja mantener los costos en el menor nivel posible, independientemente del nivel de ingresos por ventas. Esta medida permite generar el mayor nivel de valor agregado en la empresa. Si se toma como materia de ilustración el minicaso referido a 


\section{Minicaso: El dilema de los socios, ¿cuánto dura un activo?}

El modelo de mínimo costo

La compañía Equip compra una máquina en un valor inicial de \$ 10 000. La máquina debe ser trabajada, intensivamente, las 24 horas del día. Transcurren unos meses de uso y el gerente observa que los costos de operación y mantenimiento se elevan gradualmente. Entonces, surge la inquietud acerca de si es mejor comprar una nueva máquina o si se debe mantener dicho activo por un año, por dos, tres o más años.

Una máquina nueva funciona mejor y casi no requiere reparaciones, pero con el paso del tiempo disminuye su valor en el mercado de reventa y aumentan los gastos por reparación y mantenimiento. Además, durante las horas que no funciona, la máquina es reemplazada por otra.

Ahora bien, los ingresos atribuibles a la gestión del activo pueden considerarse constantes y, por lo tanto, independientes del análisis ${ }^{3}$. Dado que se está realizando el análisis hoy, conociendo el valor actual de la máquina, para futuros reemplazos puede suponerse que este valor no se modifica ${ }^{4}$. Adicionalmente, el costo de capital se considera constante en el tiempo.

Por el momento, el gerente posee la siguiente información: al finalizar el primer año, el valor residual o valor de reventa del activo es $\$ 7728$, mientras que para el segundo año es de $\$ 6118$ y para el tercer año se reduce a \$ 4941 . Mientras la máquina se conserve en la empresa, se deben asumir los costos de operación y mantenimiento, los cuales ascienden a \$2 200 el primer año, a $\$ 2370$ el segundo y a $\$ 2552$ el tercero. continuación, se sabe que todo activo debe ser analizado bajo la premisa que los costos totales incluyen tanto los costos de inversión como los costos de operación y mantenimiento. Al revisar una inversión en activos, se observa que, generalmente, existe un intercambio inverso entre los costos que implica la inversión misma frente a los costos que supone operar y mantener dichos activos.

$\mathrm{CT}=$ Costo de inversión + Costo de $\mathrm{O}$ y $\mathrm{M}$

Donde

O: Operación.

M: Mantenimiento.

Para poder analizar el ejemplo del minicaso, se emplea el concepto de 'costo anual equivalente' (CAE), que significa que cada uno de los términos debe ser señalado en términos anuales:

$$
@ \mathrm{CT}=@ \mathrm{Inv}+@ \mathrm{COyM}
$$

En adelante, se separará el flujo de inversiones del flujo de costos de operación y mantenimiento, para finalmente sumar ambos flujos o costos anuales equivalentes, a fin de determinar la vida útil económica del activo. Se utiliza un costo de oportunidad del $12 \%$ anual.

4 Ver punto 4, sobre vida útil y cambio tecnológico. 


\subsubsection{Costo anual equivalente de la inversión}

Se utiliza el concepto de anualidades para tener un esquema que permita elegir, en términos comparables, entre varias alternativas. En la Figura 2 se observa, en términos de flujos, la inversión en la máquina $\left(\mathrm{I}_{0}=\$\right.$ 10000) y dos posibles casos: (1) que se decida renovarla cada año, con lo que se obtiene, como valor residual por la venta, un ingreso neto de $\$ 7$ 728; y (2) que se decida renovarla cada dos años, con lo que se obtiene un ingreso neto de \$ 6 118. ¿Cómo saber si el costo de inversión en el primer caso es mayor o menor que en el segundo? Tenemos un problema de diferente horizonte de evaluación; esto es, en el primer caso la operación termina en un solo periodo $\mathrm{y}$, en el segundo caso, en dos.

Figura 2

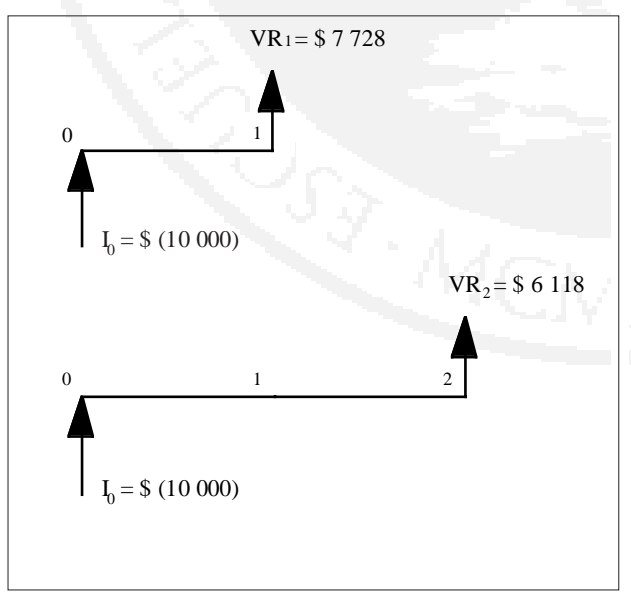

\section{Figura 3}

Esta situación puede ser resuelta si se añade una nueva operación, como se establece en la Figura 3, en la que se repite la inversión de \$ 10000 y el ingreso por valor residual, al final de primer periodo, de $\$ 7$ 728. Con esto se ha solucionado el problema del horizonte de evaluación.

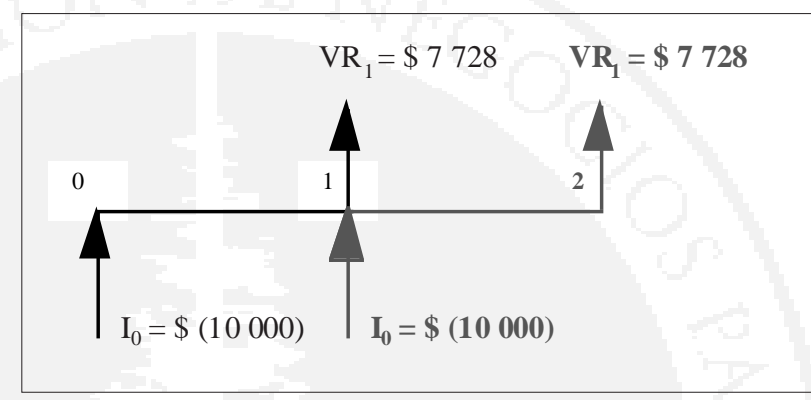

Para conocer el costo de inversión de ambas alternativas $y$, de esta forma, compararlos, se tienen dos posibilidades: (1) encontrar el valor presente de ambos flujos a dos años y (2) encontrar un costo anual equivalente. Cualquiera de las dos ofrece la respuesta; sin embargo, la segunda posibilidad tiene una ventaja: no necesita que se construyan los flujos ni sus componentes para poder utilizarla en comparar los costos de inversión entre una y otra alternativa.

El concepto del costo anual equivalente se sustenta en que las inversiones se repetirán indefinidamente; cada vez que se liquida un activo, se vuelve a invertir en uno nuevo similar. De esta forma, se considera que los reemplazos se hacen de manera perpetua.

Para llegar a la solución del minicaso, en primer lugar se debe hallar el costo 
anual equivalente de la inversión (@Inv) para cada periodo de reemplazo. Como el periodo óptimo de reemplazo es la incógnita por resolver, se ha de sensibilizar el número de periodos. Se inicia el proceso una vez que se ha encontrado el costo anual equivalente de la inversión (@ Inv $)$ de una política de reemplazos al periodo 1 .

En la Figura 4, se puede observar lo que se desea lograr: se debe cambiar el flujo compuesto por la inversión $\left(\mathrm{I}_{0}\right)$ y el valor residual al periodo $1\left(\mathrm{VR}_{1}\right)$ por un flujo único del costo anual equivalente al final del periodo 1 (@ Inv $\left.)_{1}\right)$. Se plantea el siguiente procedimiento:

1. Actualización del $\mathrm{VR}_{1}$ :

$\$ 7728 /(1+12 \%)=\$ 6900$

2. Suma en el periodo 0:

$\$-10000+\$ 6900=\$-3100$

3. @Inv al periodo 1:

$@ \operatorname{Inv}_{1}=\$-3100(1+12 \%)$

$@ \mathrm{Inv}_{1}=\$-3473$ sión al periodo 1 y se determinaba el valor neto. Sin embargo, el haber optado por el camino largo tiene un objetivo, cuya utilidad podrá apreciarse cuando se determine el costo anual equivalente de la inversión (@ $\left.\operatorname{Inv}_{2}\right)$ de una política de reemplazos al periodo 2. En la Figura 5, se aprecia lo que se espera obtener: se debe cambiar el flujo compuesto por la inversión $\left(\mathrm{I}_{0}\right)$ y el valor residual al periodo $2\left(\mathrm{VR}_{2}\right)$ por un flujo de dos costos anuales equivalentes (@Inv $)_{2}$. Se desarrolla el siguiente procedimiento:

1. Actualización del $\mathrm{VR}_{2}$ :

\$ $6118 /(1+12 \%)=\$ 4877$

2. Suma en el periodo 0:

$\$-10000+\$ 4877=\$-5123$

3. @Inv a los periodos 1 y 2 :

$@ \operatorname{Inv}_{2}=\operatorname{Pago}(12 \%, 2, \$ 5123)^{5}$

$@ \operatorname{Inv}_{2}=\$-3031$

De esta forma, se puede concluir en que el costo anual equivalente de la inversión de una política de reemplazos cada

\section{Figura 4}

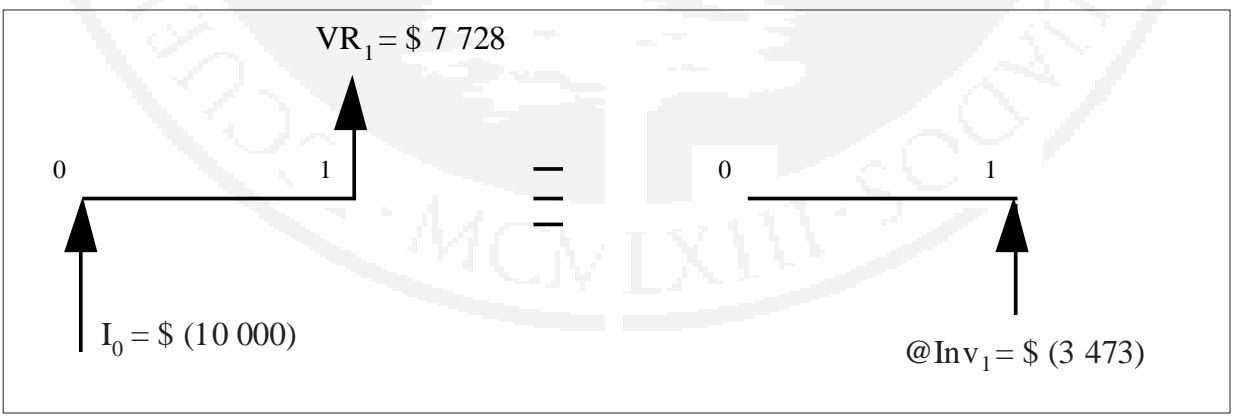

Se puede sostener que idéntica respuesta, y de forma más directa, pudo haberse alcanzado si se llevaba la inver-
5 Se utiliza la fórmula de 'pagos iguales' bajo el formato de las hojas de cálculo. Pago $(\mathrm{k} \%, \mathrm{n}, \mathrm{I})$ o PMT (k\%,n,I), en las hojas en inglés (pago o payment). 
Figura 5

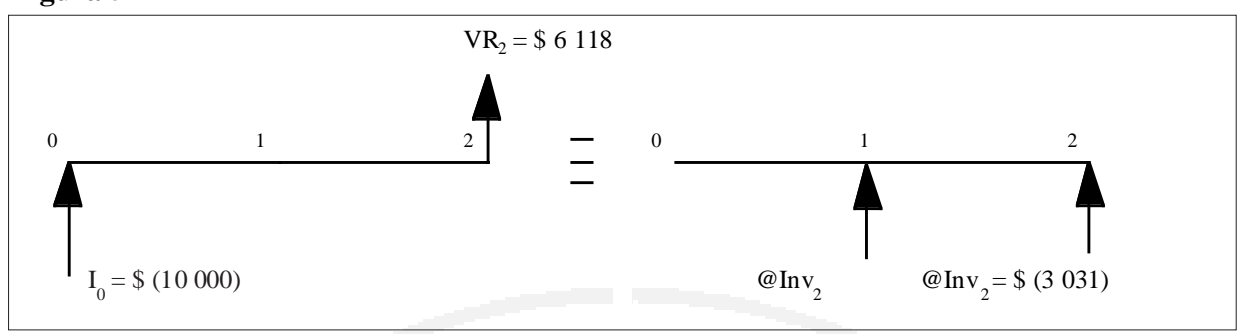

Figura 6

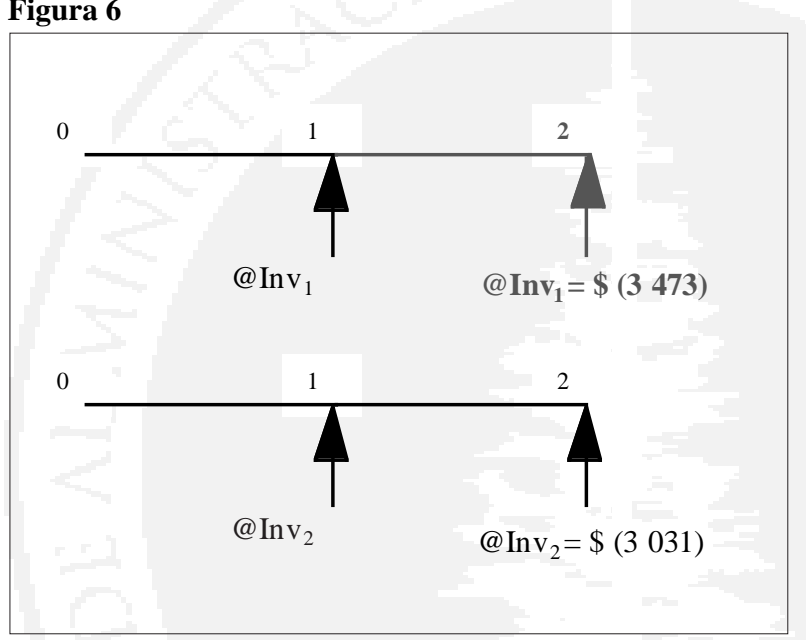

la inversión ( @ Inv 3 ) de una política de reemplazos al periodo 3. En la Figura 7 se observa lo que se espera obtener: se debe cambiar el flujo compuesto por la inversión $\left(\mathrm{I}_{0}\right)$ y el valor residual al periodo $3\left(\mathrm{VR}_{3}\right)$ por un flujo de tres costos anuales equivalentes (@ Inv $v_{3}$.Se desarrolla el siguiente procedimiento:

1. Actualización del $\mathrm{VR}_{3}$ :

$\$ 4941 /(1+12 \%)^{3}=\$ 3517$

2. Suma en el periodo 0: $\$-10000+\$ 3517=\$-6483$

que el correspondiente a una política de reemplazos cada periodo anual ( $@ \operatorname{Inv}_{1}=$ $\$$-3 473). En la Figura 6, se presenta el sustento de por qué estas anualidades del costo de la inversión son equivalentes y por qué, en el caso del reemplazo en cada periodo anual, se repite para igualar el horizonte de evaluación respecto a un reemplazo cada dos periodos. Si bien se utilizan los flujos para mostrar la consistencia del análisis, en realidad se puede realizar la comparación directamente con las anualidades.

Para consolidar la metodología, se determina el costo anual equivalente de
3. @Inv a los periodos 1, 2 y 3: $@ \operatorname{Inv}_{3}=$ Pago $(12 \%, 3, \$ 6483)$ $@ \operatorname{Inv}_{3}=\$-2699$

En la Figura 8 se comparan los costos anuales equivalentes de la inversión resultantes de políticas de reemplazo a uno, dos y tres periodos. Nótese que la metodología se sustenta en la construcción de flujos con horizonte de evaluación equivalente. Se observa que, analizando únicamente el flujo de inversiones, conviene mantener el activo durante tres años, por presentarse los menores costos anuales equivalentes de la inversión (@Inv); 
Figura 7

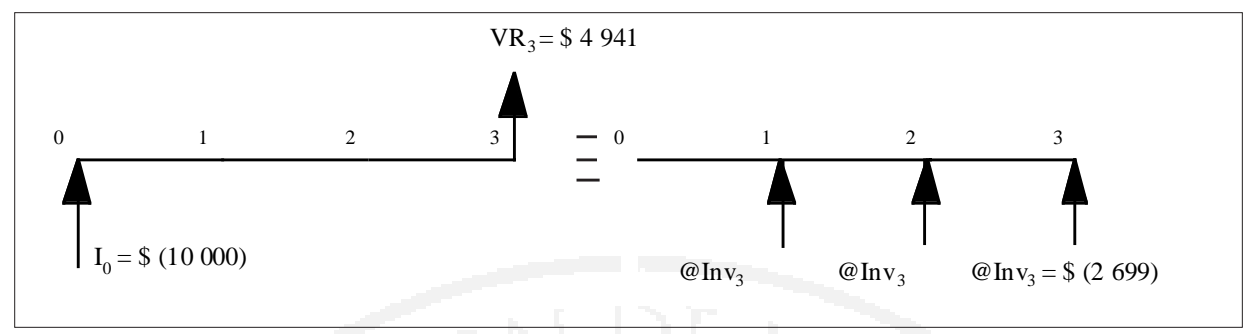

\section{Figura 8}

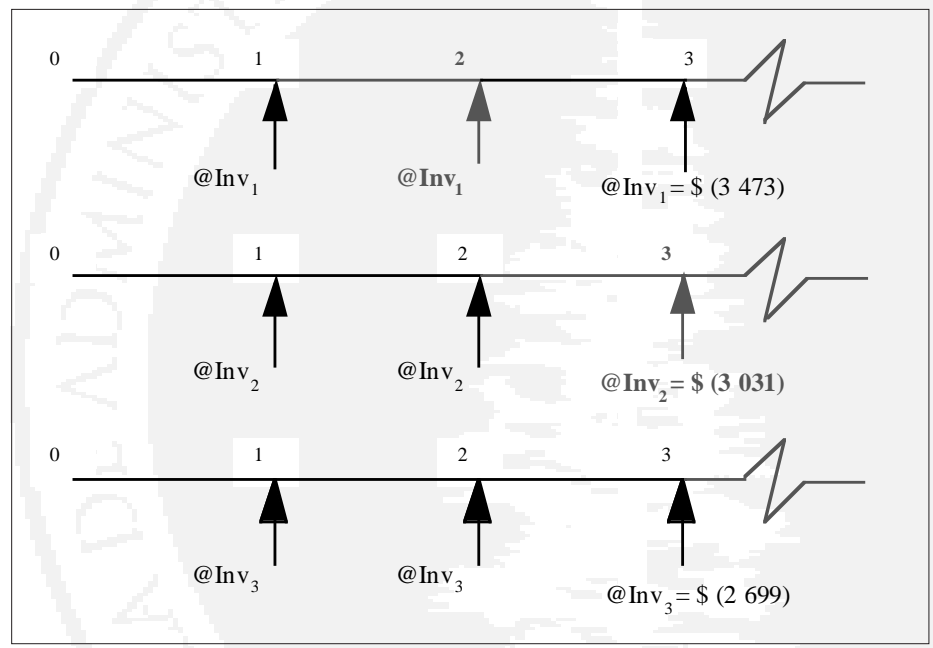

tenimiento de la máquina en dos posibles casos: (1) que se decida renovarla cada año, por lo que se pagaría $\$ 2200$ al año, y (2) que se decida renovarla cada dos años, por lo que se pagaría $\$ 2200$ el primer año y $\$ 2370$ el segundo. ¿Cómo saber si el costo de operación y mantenimiento en el primer caso es mayor o menor que en el segundo? Se está, nue-

sin embargo, el análisis no es completo si no se analiza el otro componente de los costos de un activo: los costos de operación y mantenimiento.

\subsubsection{Costo anual equivalente de la operación y mantenimiento}

De forma similar, se utiliza el concepto de anualidades, con la finalidad de comparar y elegir entre varias alternativas. $\mathrm{Si}$ se analiza la Figura 9, se tiene, en términos de flujos, los costos de operación y man- vamente, frente a un problema de diferente horizonte de evaluación.

La situación puede ser resuelta si se añade una nueva operación, tal como se establece en la Figura 10, en la que se ha repetido el costo de operación y mantenimiento del periodo $1\left(\mathrm{COyM}_{1}=\right.$ $\$-2$ 200). Con esto se ha solucionado, de la misma manera que para la inversión, el problema del horizonte de evaluación.

Se debe encontrar, luego, el costo anual equivalente de operación y mante- 
Figura 9

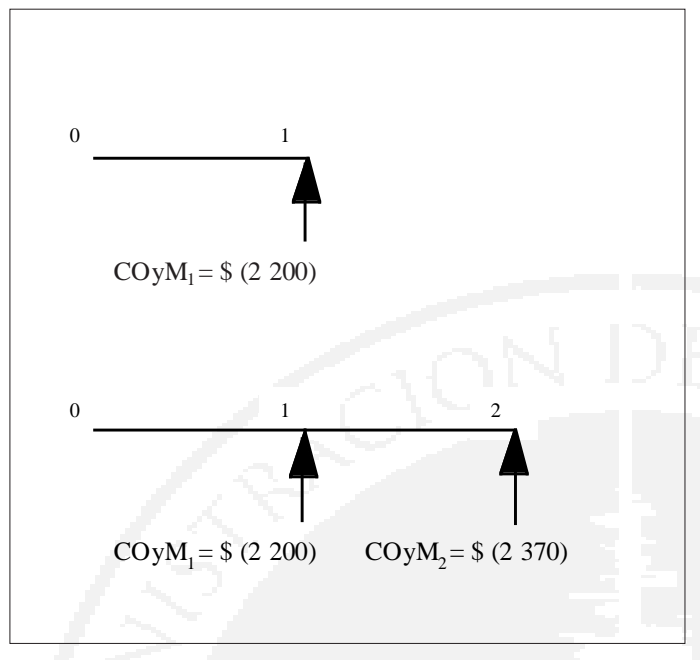

Figura 10

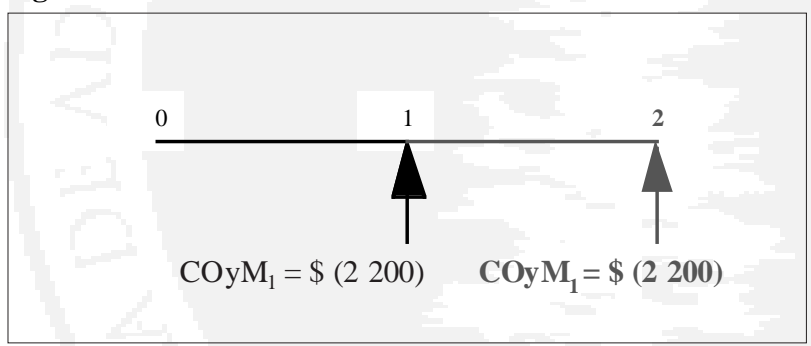

zos al periodo 1. En la Figura 11 se percibe lo que se quiere lograr: se debe cambiar el flujo del costo de operación y mantenimiento del periodo $1\left(\mathrm{COyM}_{1}\right)$ por un flujo único del costo anual equivalente al final del periodo 1 (@ $\left.\mathrm{COyM}_{1}\right)$. Como puede apreciarse, no se necesita de ningún cálculo (@ $\mathrm{COyM}_{1}=\$-2$ 200).

También se puede determinar el costo anual equivalente de la operación y mantenimiento ( $\left.@ \mathrm{COyM}_{2}\right)$ de una política de reemplazos al periodo 2. En la Figura 12, se observa lo esperado: cambiar los flujos de los periodos 1 y 2 $\left(\mathrm{COyM}_{1}\right.$ y $\left.\mathrm{COyM}_{2}\right)$ por un flujo de dos costos anuales equivalentes(@ $\mathrm{COyM}_{2}$ ); de esta forma, se obtiene lo siguiente:

1. Actualización del $\mathrm{COyM}_{2}$ :

$\$ 2200 /(1+12 \%)+\$ 2370 /$ $(1+12 \%)^{2}=\$ 3854$ nimiento (@COyM) para cada periodo de reemplazo. Como el periodo óptimo de reemplazo vuelve a ser la incógnita por resolver, se ha de sensibilizar otra vez el número de periodos. Se inicia el proceso encontrando el costo anual equivalente de la operación y mantenimiento (@ $\left.\mathrm{COyM}_{1}\right)$ de una política de reempla-

\section{Figura 11}

2. @COyM a los periodos 1 y 2 : $@ \mathrm{COyM}_{2}=$ Pago $(12 \%, 2, \$ 3$ 854) @ $\mathrm{COyM}_{2}=\$-2280$

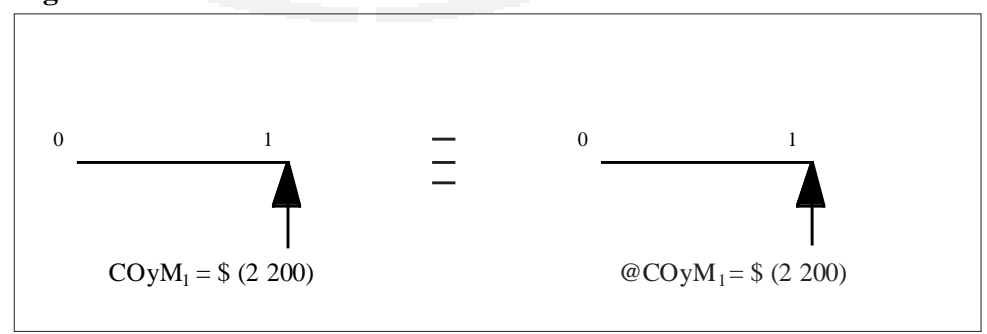


Obsérvese que el @ COyM $=-\$ 2280$ es un promedio financiero entre el $\mathrm{COyM}_{1}$ $\mathrm{y}$ el $\mathrm{COyM}_{2}$.

Se puede concluir en que el costo anual equivalente de operación y mantenimiento de una política de reemplazos en cada reemplazo cada dos periodos. Se utilizan flujos para mostrar la consistencia del análisis, aunque es posible comparar directamente con anualidades.

Para consolidar la metodología, se determina el costo anual equivalente de

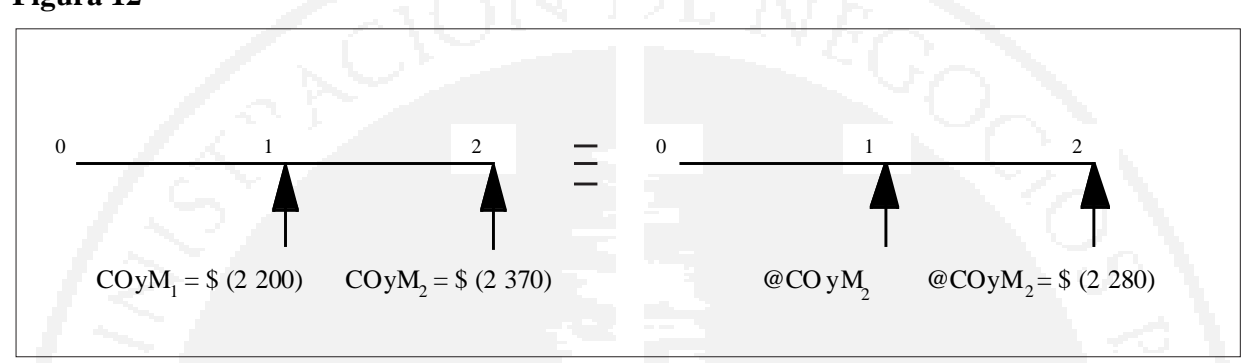

periodo anual (@ $\mathrm{COyM}_{1}=\$-2$ 200) es menor que el correspondiente a una política de reemplazos cada dos periodos (@ $\mathrm{COyM}_{2}=\$-2$ 280). La Figura 13 presenta el porqué estas anualidades son equivalentes: si se reemplaza en cada periodo anual, se repite el flujo para igualar el horizonte de evaluación respecto a un la operación y mantenimiento (@ $\mathrm{COyM}_{3}$ ) de una política de reemplazos al periodo 3. En la Figura 14 se puede ver lo esperado: cambiar los flujos de los periodos 1 , 2 y $3\left(\mathrm{COyM}_{1}, \mathrm{COyM}_{2}\right.$ y $\left.\mathrm{COyM}_{3}\right)$ por un flujo de tres costos anuales equivalentes (@COyM3), lo que se logra con el siguiente procedimiento:

\section{Figura 13}

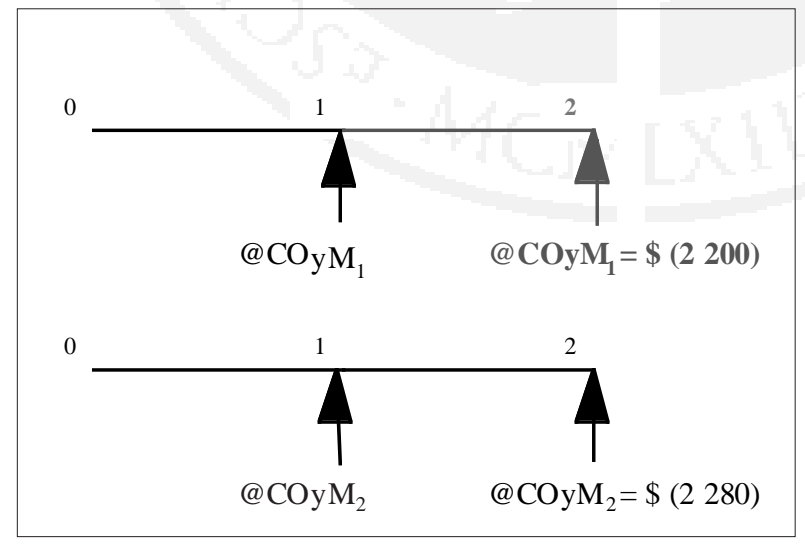

1. Actualización del $\mathrm{COyM}_{3}$ : $\$ 2200 /(1,12)+\$ 2370 /(1,12)^{2}$ $+\$ 2552 /(1,12)^{3}=\$ 5670$

2. @COyM a los periodos 1,2 y 3:

@ $\mathrm{COyM}_{3}=\operatorname{Pago}(12 \%, 3$, $\$ 5670$ ) $@ \mathrm{COyM}_{3}=\$-2361$.

En la Figura 15 se comparan los costos anuales equivalentes de operación y mantenimiento resultantes de políticas de reemplazo a uno, dos y tres periodos. Nótese 
Figura 14

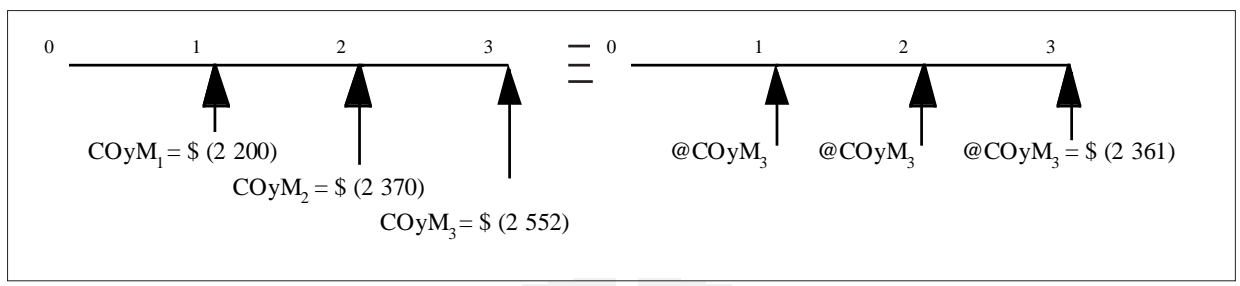

que la metodología se sustenta en la construcción de flujos con horizonte de evaluación equivalente. Se observa que, analizando únicamente el flujo de operación y mantenimiento, conviene mantener el activo durante un solo año, por presentarse los menores costos anuales equivalentes de operación y mantenimiento (@COyM).

\section{Figura 15}

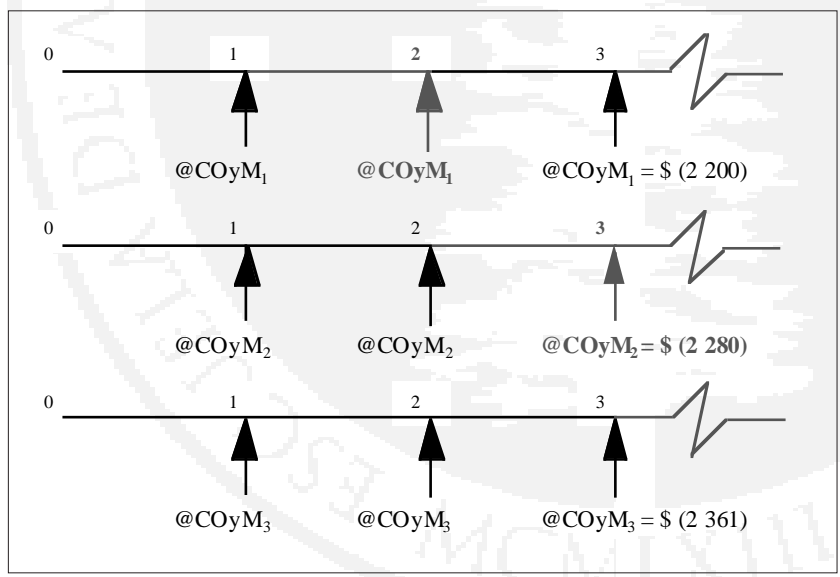

\subsubsection{Costo anual equivalente total}

El análisis completo comprende los dos componentes de los costos de un activo: los costos de inversión y los costos de operación y mantenimiento, como se observa en la Figura 16, para una política de reemplazo a un periodo.
Finalmente, para cada alternativa se halla el @CT sumando el @Inv y el @COyM.

- Para una política de reemplazo a un periodo: $@ \mathrm{CT}=\$ 3473+\$ 2200=\$ 5673$

- Para una política de reemplazo a dos periodos:

$@ \mathrm{CT}=\$ 3031+\$ 2280=\$ 5312$

- Para una política de reemplazo a tres periodos:

$$
\begin{aligned}
& @ \mathrm{CT}=\$ 2699+\$ 2361 \\
& =\$ 5060
\end{aligned}
$$

Una vez hallados los @CT que se muestran en la Figura 17, se puede responder a la interrogante de los socios: una política de reemplazo a tres periodos otorga costos anuales equivalentes totales menores.

\subsection{Extensión del minicaso a ' $n$ ' periodos}

El resultado anterior es válido para la decisión entre tres periodos anuales y se puede extender a más periodos para determinar la vida útil económica del activo y la mejor política de reemplazo de activos. Si se incluyeran todos los costos de operación y mantenimiento y los valores 
Figura 16

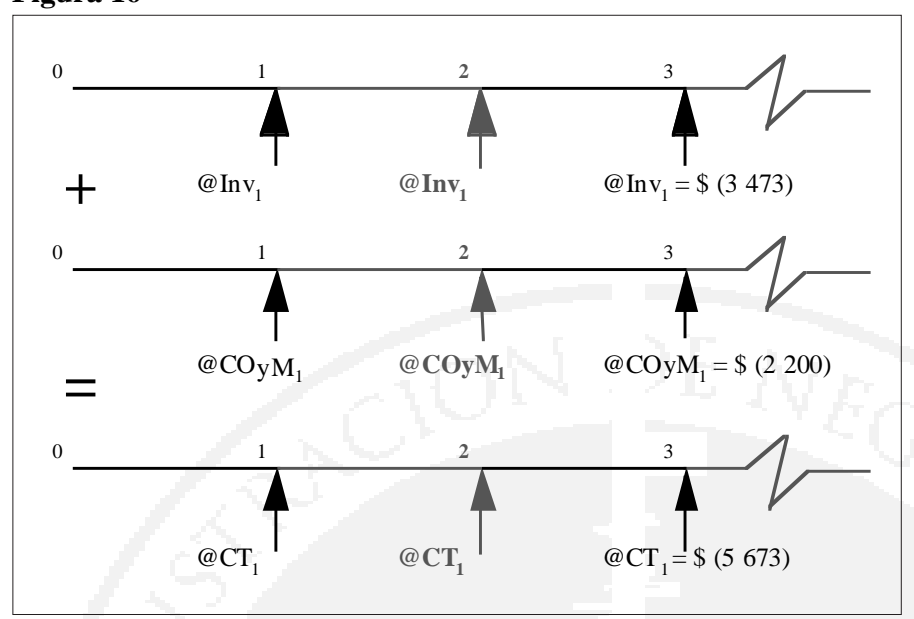

Figura 17

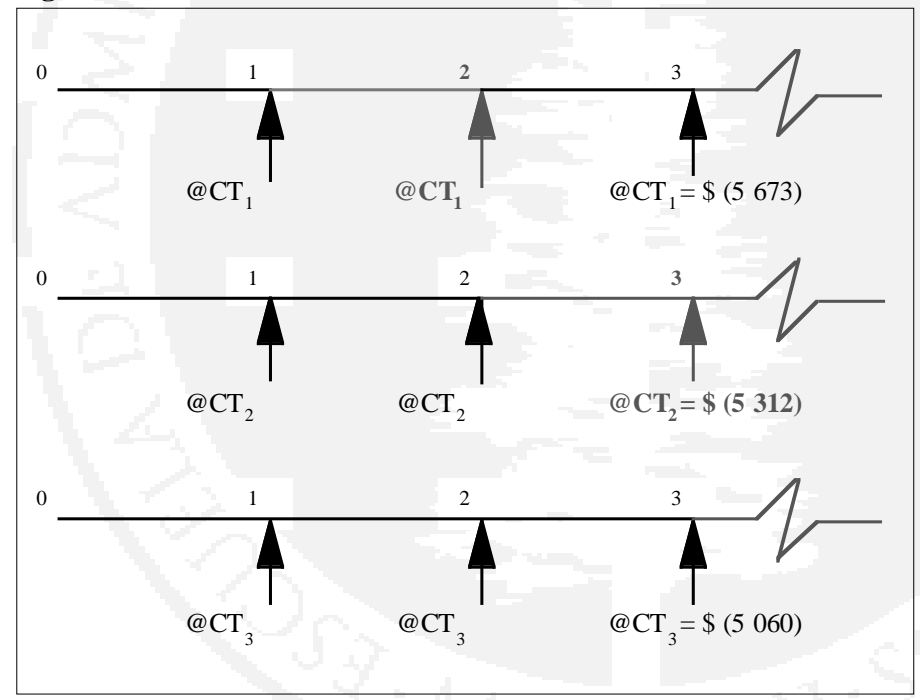

operación y mantenimiento para cada año; estos datos proceden del mercado o de los archivos históricos de la empresa y varían con el paso del tiempo.

El valor residual, que es menor para cada periodo, se actualiza al costo de oportunidad de $12 \% \mathrm{y}$ se suma a la inversión inicial para, luego, convertir el resultado a pagos iguales anuales para obtener el costo anual equivalente de la inversión(@Inv).

\section{El COyM suma} los costos de operación y de mantenimiento previstos para cada periodo. Se actualiza esta suma al costo de oportunidad de $12 \%$ para cada periodo, desde el periodo inicial, y este valor se convierte a pagos

residuales correspondientes, los pasos por seguir para llegar a la solución serían los mismos que los presentados antes; sin embargo, el procedimiento se vuelve tedioso, tanto en su explicación como en su entendimiento.

En la Figura 18 se presentan las cifras finales. Se han tomado como datos los valores residuales y los costos de iguales anuales, con lo que obtiene el costo anual equivalente de operación y mantenimiento (@COyM).

La suma de ambos costos anuales equivalentes es el costo anual equivalente total (@CT) que sirve para determinar la vida útil económica del activo. En la Figura 18, se aprecia que el @ CT mínimo se produce durante el año 6 , que es cuando 


\section{Figura 18}

Determinación de la vida útil económica

Activos fijos no relacionados con ingresos

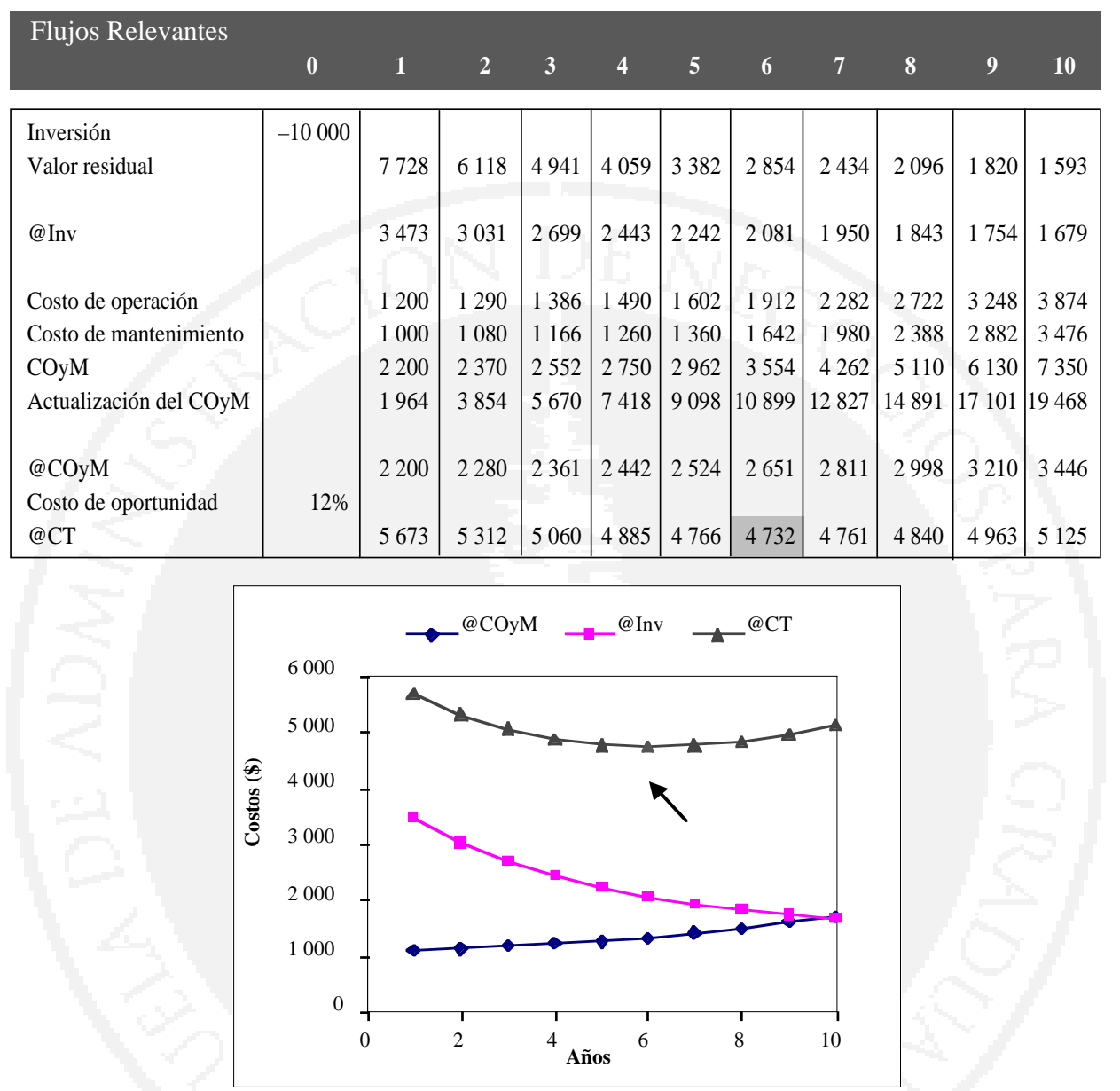

se debe liquidar el activo y reemplazarlo por otro; de lo contrario, su permanencia en la empresa ya no es beneficiosa. Por lo tanto, este activo tiene una vida útil económica de seis años.

\section{Vida útil y cambio tecnológico}

En la sección 3 de este trabajo, se presupone que el activo se renueva por otros activos de idénticas características. En el supuesto del futuro como una extensión del presente, pero de un presente en constante evolución y no estático, la hipótesis de un activo por otro idéntico no se sustenta con lo que pasa en la realidad, porque hace abstracción del progreso tecnológico actual.

Con el transcurso del tiempo, los activos mejoran técnicamente; se ofrecen a me- 
nores costos debido a la mayor eficiencia y productividad. Para que la empresa siga compitiendo en el mercado, debe sustituir progresivamente los activos antiguos por los últimos modelos existentes en el momento en que se plantea la necesidad de renovar.

Todo activo se deprecia con el tiempo, no sólo física sino también técnicamente, y experimenta, con relación al último modelo aparecido en el mercado, una inferioridad de servicio. La inferioridad de servicio o 'depreciación por obsolescencia tecnológica' crece linealmente en función del tiempo, por lo que la presión por reemplazar el activo crece de manera continua.

No obstante, en la construcción del modelo de mínimo costo se asume reemplazo de activos idénticos basado en una premisa implícita. En el largo plazo, se espera que los precios de los productos o servicios se abaraten, al mismo tiempo que caen los costos de producción, mientras que los volúmenes de venta aumentan. Con esta combinación, los márgenes de utilidad para el negocio tienden a mantenerse más o menos constantes en el largo plazo, aunque se espera que se incrementen. Por esta razón, es aceptable simular el reemplazo de activos a los valores actuales, que son los únicos certeramente disponibles en la mayoría de los casos.

Sin embargo, es razonable profundizar el análisis, porque el valor residual puede resultar menor al supuesto o porque el COyM supuesto resulta ser inferior en la realidad. Lo primero ocurre debido a que la innovación tecnológica puede depreciar los activos antiguos; y lo segundo, a causa de que la competencia puede obligar a bajar los precios de los productos reduciendo los márgenes y presionando hacia una reducción de costos. Esto supone que, si se quieren incorporar los efectos de la innovación tecnológica, se puede suponer que los valores residuales se deteriorarán con el tiempo y que el COyM, relativamente, será más alto. Después de señalar esta posición conservadora, se puede sortear el problema tecnológico. Se puede afectar la inversión con una razón de reducción en el tiempo, de acuerdo con la experiencia y las perspectivas que se tengan con los precios de los activos. En este caso, la forma de solucionar el problema parte del esquema de perpetuidades con índices de crecimiento o decrecimiento.

En la Figura 19, se halla el valor de la inversión, si esta decrece a una tasa constante $\mathrm{g} \%$ (tendría valores negativos como, por ejemplo, $-5 \%$ ), que igualmente afecta al valor residual. Se sigue el siguiente procedimiento:

a) Se encuentra el valor presente de las inversiones, considerando que decrecen a una tasa constante $\mathrm{g} \%$ : $\mathrm{VP}(\mathrm{Inv})=$ $\mathrm{I}_{0}+\mathrm{I}_{0}\left(1+\mathrm{g}_{\mathrm{n}}\right) /\left(\mathrm{k}_{\mathrm{n}}-\mathrm{g}_{\mathrm{n}}\right)$.

b) Se halla el valor presente del valor residual, considerando que decrece igualmente a una tasa constante $\mathrm{g} \%$ : VP(VR) $=\mathrm{VR} /\left(\mathrm{k}_{\mathrm{n}}-\mathrm{g}_{\mathrm{n}}\right)$.

c) Tómese en cuenta que el k considerado debe corresponder al periodo de reemplazo $\mathrm{n}$, determinándose de la siguiente manera: $\mathrm{k}_{\mathrm{n}}=(1+\mathrm{k})^{\mathrm{n}}-1$.

d) De igual forma, la tasa de crecimiento corresponde al periodo de reemplazo: $g_{\mathrm{n}}=(1+\mathrm{g})^{\mathrm{n}}-1$. 


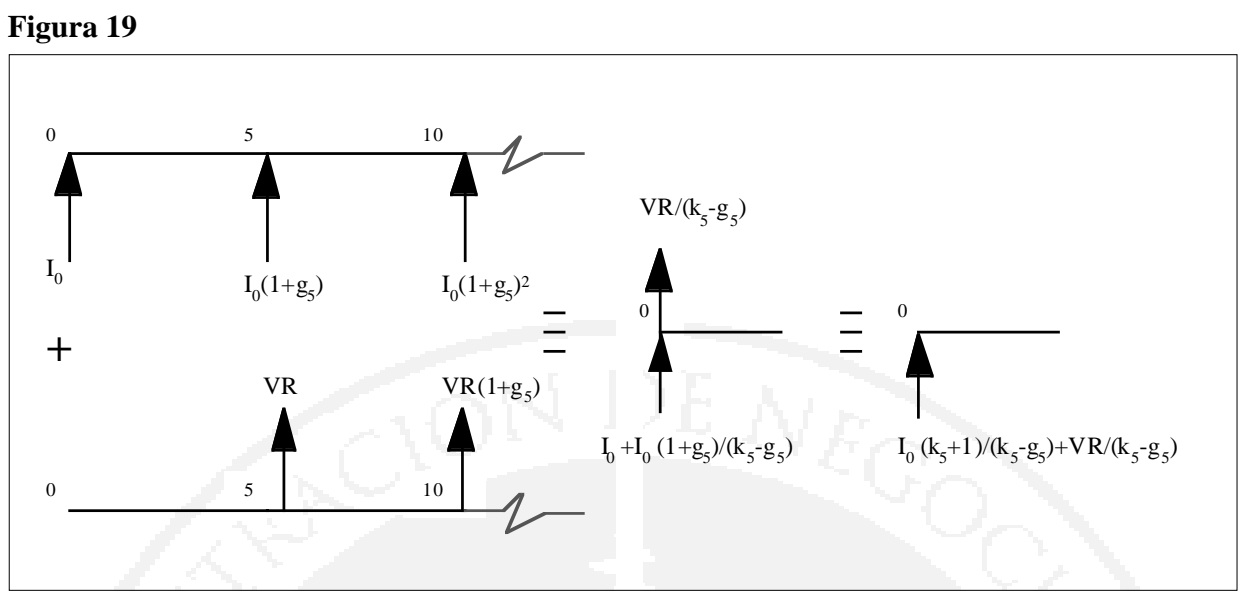

Figura 20

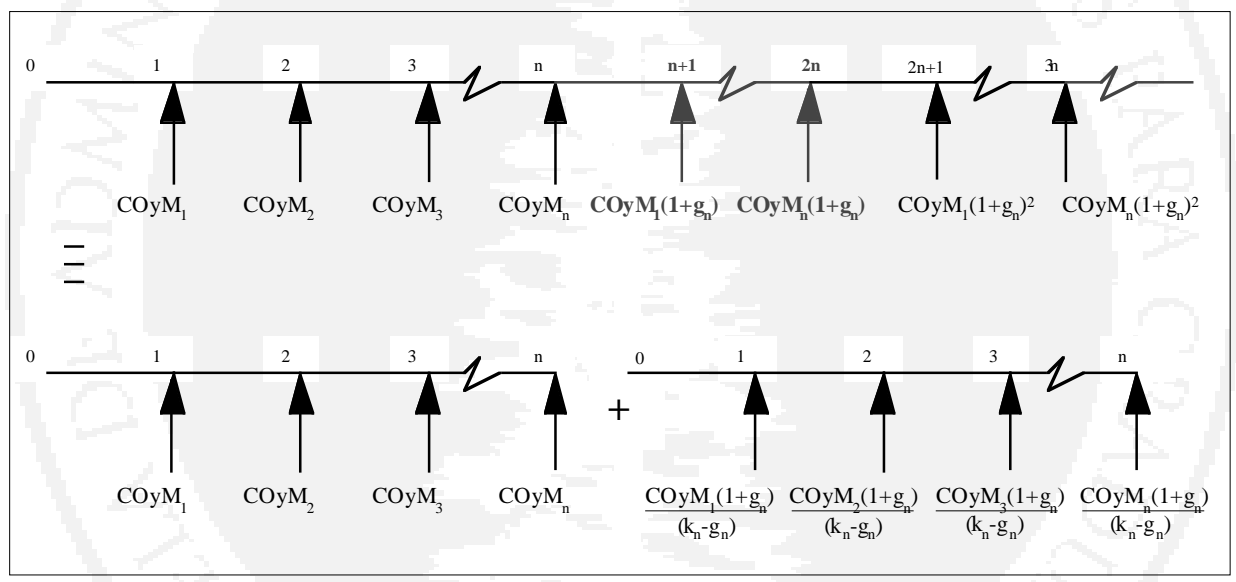

e) El valor presente de la inversión neta, correspondiente al periodo n, está determinado por la siguiente expresión:

$$
\operatorname{VPN}(\operatorname{Inv})=\frac{\mathrm{I}_{\mathrm{O}}\left(\mathrm{k}_{\mathrm{n}}+1\right)+\mathrm{VR}}{\left(\mathrm{k}_{\mathrm{n}}-\mathrm{g}_{\mathrm{n}}\right)}
$$

En la Figura 20 se muestran los costos de operación y mantenimiento para una política de reemplazo a $\mathrm{n}$ periodos, donde el COyM decrece a una tasa constante $\mathrm{g}_{\mathrm{n}}$ entre cada periodo de reemplazo. Para en- contrar el valor presente neto del COyM, se sigue el siguiente procedimiento:

a) Se encuentra el valor presente individual de cada uno de los COyM, considerando que decrecen a una tasa constante $\mathrm{g} \%: \mathrm{VP}\left(\mathrm{COyM}_{\mathrm{i}}\right)=\left[\mathrm{COyM}_{\mathrm{i}}+\right.$ $\left.\operatorname{COyM}_{i}\left(1+g_{n}\right) /\left(k_{n}-g_{n}\right)\right] /[1+k]^{i}$.

b) Como en el caso anterior, el $\mathrm{k}_{\mathrm{n}} \mathrm{y}$ la tasa $g_{n}$ corresponden al periodo de reemplazo $\mathrm{n}$. 
c) El valor presente de los COyM está determinado por la siguiente expresión:

$$
\operatorname{VPN}(\operatorname{COyM})=\sum_{i=1}^{n} \frac{\operatorname{COyM}_{\mathrm{i}}\left(\mathrm{k}_{\mathrm{n}}+1\right)}{\left(\mathrm{k}_{\mathrm{n}}-\mathrm{g}_{\mathrm{n}}\right)(1+\mathrm{k})^{\mathrm{i}}}
$$

Finalmente, se poseen los valores presentes de los costos de inversión y el
COyM. De forma similar, como en los casos anteriores, se realiza la suma de ambos conceptos para cada periodo de reemplazo. Se elige como vida útil aquella que otorgue el menor valor presente de la suma de ambos costos. Se evalúa, de acuerdo con la aplicación, cuál de los dos métodos es el más razonable. 


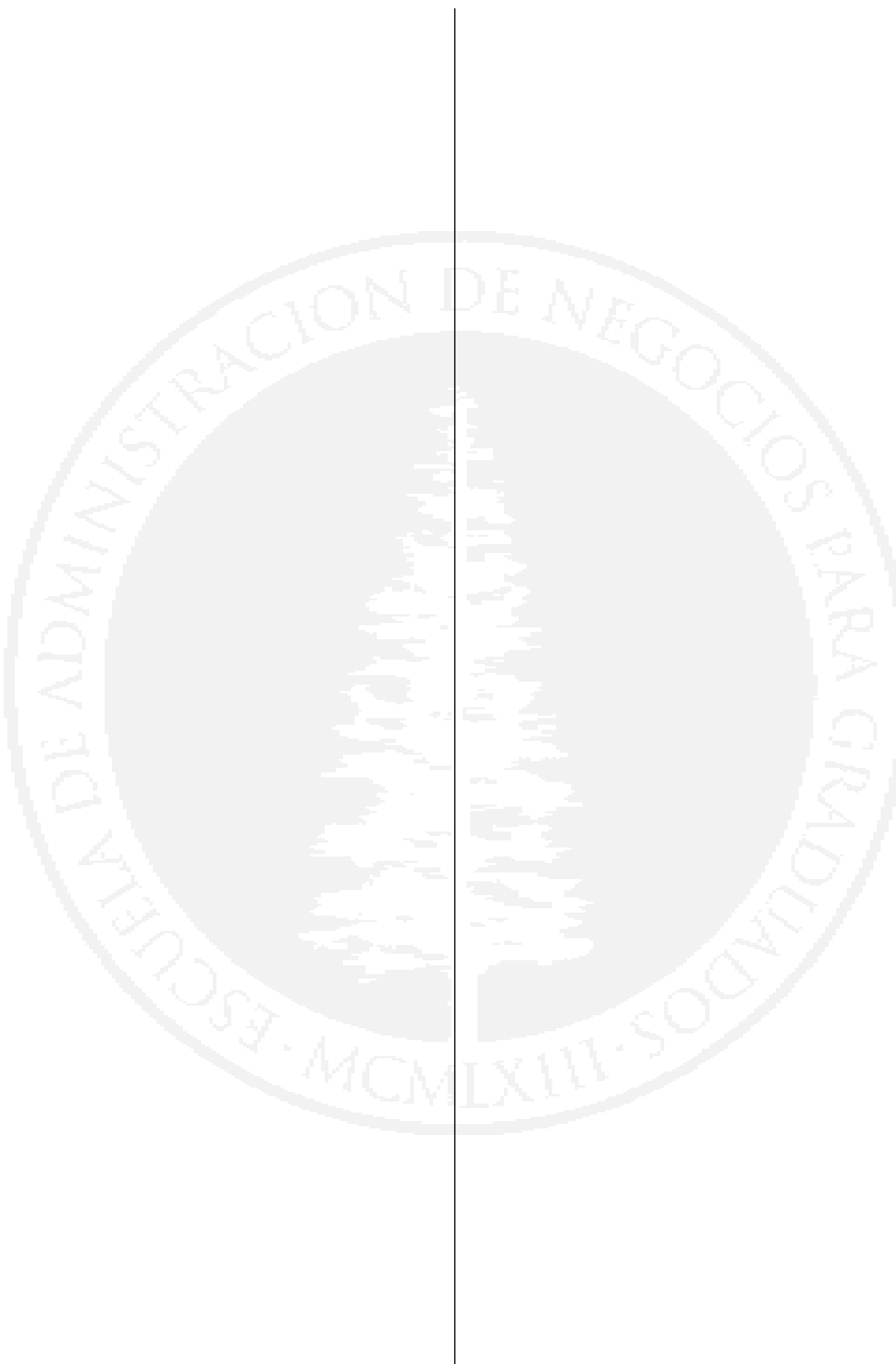

